\title{
INDEX TO VOLUME 58
}

Author

ARNOLD, ANTON \&

NIER, FRANCIS

BOHMAN, JAN \&

Fröberg, CARL-ERIK

DE BOOR, CARL \&

RON, AMOS

Bramble, James H. \&

Pasciak, Joseph E.

BROEZE, JAN \&

van DaAlen, Edwin F. G.

BROEZE, JAN

Chaladus, Stefan

Chan, Raymond H. \&

Yeung, Man-Chung

Chen, C., Thomé, V. \&

WAHLBIN, L. B.

Chui, C. K., Stöckler, J. \&

WARD, J. D.

Colbourn, Charles J.,

Magliveras, SPYros S. \& Mathon, Rudolf A.

Costabel, M., Penzel, F. \& SCHNEIDER, $R$.

CouzY, W.

Crisci, M. R., Russo, E. \& Vecchio, A.

van DaAlen, Edwin F. G., BROEZE, JAN \& VAN GROESEN, EMBRECht

van DaAlen, Edwin F. G.

Dawson, Clint N. \& DUPONT, TODD F.

Del Corso, Ilaria

Di Natale, M., Gotusso, L., Pavani, R. \& Roux, D.

DÖRFLER, $W$.

DUBNer, HARVEY

DUPONT, TODD F.

DURÁN, RICARDO \&

Liberman, Elsa

Edelman, Alan
Title

Page

Numerical analysis of the deterministic particle method applied to the Wigner equation . . . . . . . . . . . . 645

The $\Gamma$-function revisited: Power series expansions and realimaginary zero lines $\ldots \ldots \ldots \ldots \ldots$

Computational aspects of polynomial interpolation in several variables . . . . . . . . . . . . . . 705

The analysis of smoothers for multigrid algorithms . . . 467

Radiation boundary conditions for the two-dimensional wave equation from a variational principle . . . . . .

See: van DaAlen, Edwin F. G., Broeze, Jan \& vaN Groesen, EMbrecht . . . . . . . . . .

On the densest lattice packing of centrally symmetric octahedra . . . . . . . . . . . . . . .

Circulant preconditioners for Toeplitz matrices with positive continuous generating functions $\ldots \ldots \ldots \ldots$

Finite element approximation of a parabolic integrodifferential equation with a weakly singular kernel ...

A Faber series approach to cardinal interpolation ... . 255

Transitive Steiner and Kirkman triple systems of order 27

Error analysis of a boundary element collocation method for a screen problem in $\mathbf{R}^{3} \ldots \ldots \ldots \ldots \ldots \ldots$. . . . .

See: van Der Houwen, P. J., Sommeijer, B. P. \& Couzy,

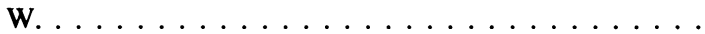

Stability results for one-step discretized collocation methods in the numerical treatment of Volterra integral equations

Variational principles and conservation laws in the derivation of radiation boundary conditions for wave equations. . . . . . . . . . . . . . 55

See: Broeze, JAN \& van DaAlen, Edwin F. G. . . . . . 73

Explicit/implicit conservative Galerkin domain decomposition procedures for parabolic problems . . . . . . . 21

Factorization of prime ideal extensions in number rings . 849

On the choice of parameter in a method for the inversion of Fourier series. . . . . . . . . . . . . . 737

Hierarchical bases for elliptic problems . . . . . . . . . 513

Partitions approximated by finite cosine-series . . . . . 729

See: Dawson, Clint N. \& Dupont, Todd F. . . . . . . 21

On mixed finite element methods for the Reissner-Mindlin plate model . . . . . . . . . . . . . . . . 561

On the distribution of a scaled condition number $\ldots \ldots 185$ 
Author

EICHENAUER-HERRMANN, JÜRGEN \& NIEDERREITER, HARALD

Elliott, Charles M. \& LARSSON, STIG

ERNVALL, R. \& MetsänKylä, T.

FröberG, CARL-ERIK

GAO, F.

GatermanN, Karin, HOFFMANN, Christoph \& Opfer, Gerhard

VAN DER GeER, Gerard, SCHOOF, RENE \& VAN DER Vlugt, MARCel

Glassey, R. T.

\section{GOLDFELD, DoRIAN}

Gotusso, L.

GouvêA, F. \& MAZur, B.

Grady, Michael \&

NewMan, MorRIS

vaN GROESEN, EMBREChT

GUO, BEN-Yu \&

MILLER, J. J. H.

HOFFMANN, CHRISTOPH

Hong, Y. P. \& Pan, C.-T.

Hou, Thomas Y.

VAN DER HOUWEN, P. J., SOMMEIJER, B. P. \& COUZY, W.

HUNDSDORFER, WILlEM

JakUbec, Stanislav \& MARKo, FrantiśeK

KECHKAR, NASSERDINE \& SILVESTER, DAVID

KEIPER, J. B.

LARSSON, STIG

LEHMAN, J. LARRY

LEVILLAIN, VINCENT

LIBERMAN, ELSA

Magliveras, Spyros S.

MARKo, František
Title

Page

Lower bounds for the discrepancy of inversive congruential pseudorandom numbers with power of two modulus . .

775

Error estimates with smooth and nonsmooth data for a finite element method for the Cahn-Hilliard equation . . . 603

Computation of the zeros of $p$-adic $L$-functions $\ldots \ldots \quad 815$

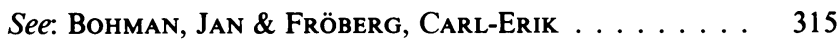

See: Wasilkowski, G. W. \& GaO, F. . . . . . . . . . . 285

Explicit Faber polynomials on circular sectors . . . . . . 241

Weight formulas for ternary Melas codes . . . . . . . . .

Convergence of an energy-preserving scheme for the Zakharov equations in one space dimension . . . . . .

On the computational complexity of modular symbols . .

See: Di Natale, M., Gotusso, L., Pavani, R. \& Roux, D. 737

Families of modular eigenforms . . . . . . . . . . . 793

Some divisibility properties of the subgroup counting function for free products . . . . . . . . . . . . . 347

See: van Daalen, Edwin F. G., Broeze, Jan \& van Groesen, EMbrecht . . . . . . . . . . . .

Iterative and Petrov-Galerkin methods for solving a system of one-dimensional nonlinear elliptic equations . . . . .

See: Gatermann, Karin, Hoffmann, Christoph \& Opfer,

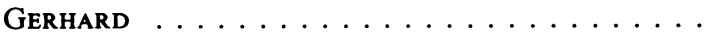

Rank-revealing $\mathrm{QR}$ factorizations and the singular value decomposition . . . . . . . . . . . .

A new desingularization for vortex methods . . . . . . . .

Embedded diagonally implicit Runge-Kutta algorithms on parallel computers . . . . . . . . . . .

Unconditional convergence of some Crank-Nicolson LOD methods for initial-boundary value problems . . . . .

Witt equivalence classes of quartic number fields . . . . . . 35

355

Analysis of locally stabilized mixed finite element methods for the Stokes problem . . . . . . . . . . . . . .

Power series expansions of Riemann's $\xi$ function . . . 765

See. Elliott, Charles M. \& Larsson, Stig . . . . . . 603

Levels of positive definite ternary quadratic forms . . . . 399

Eigenvalue approximation by a mixed method for resonant inhomogeneous cavities with metallic boundaries.... 11

See. Durán, Ricardo \& Liberman, Elsa . . . . . . . . 561

See: Colbourn, Charles J., Magliveras, Spyros S. \& Mathon, Rudolf A. . . . . . . . . . . . . 441

See: Jakubec, Stanislav \& Marko, Frantisek . . . . . 355 
Author

Mathon, Rudolf A.

Mayer, Daniel C.

MAZUR, B.

MetSÄNKYLÄ, T.

MILLER, J. J. H.

Newman, Morris

NiEdERREITER, HARALD

NiEDERREITER, HARALD

NIER, FRANCIS

NiIJIMA, KoICHI

Notaris, Sotirios E.

OLIVIER, M.

Opfer, Gerhard

Pan, C.-T.

Pasciak, Joseph E.

Pavani, R.

Penzel, F.

Peralta, René

Plonka, G. \& Tasche, M.

Richter, GeraRd R.

Ron, AMOs

Roux, D.

Rundell, William \&

Sacks, Paul E.

Russo, E.

Sacks, Paul E.

SChMitT, BERNhARD A.

SCHNEIDER, $R$.

SCHOOF, RENE

SCOTt, L. RIDGWAY \&

Zhang, Shangyou

SHOUP, VICTOR

SilVester, DAVID

SKoruppa, Nils-Peter

SOMMEIJER, B. P.

STÖCKLER, J.
Title

Page

See: Colbourn, Charles J., Magliveras, Spyros S. \& Mathon, Rudolf A. . . . . . . . . . . . 441

Multiplicities of dihedral discriminants . . . . . . . 831

See: Gouvêa, F. \& Mazur, B. . . . . . . . . . . . . . . 793

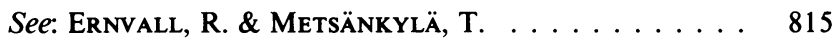

See: Guo, Ben-yu \& Miller, J. J. H. . . . . . . . . . . 531

See: Grady, Michael \& Newman, Morris . . . . . . 347

See: Eichenauer-HerRmanN, JÜrgen \& NiederReiter,

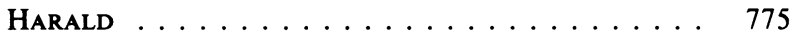

The existence of efficient lattice rules for multidimensional numerical integration . . . . . . . . . . 305

See: Arnold, Anton \& Nier, Francis . . . . . . . . 645

A posteriori error bounds for piecewise linear approximate solutions of elliptic equations of monotone type ....

On Gauss-Kronrod quadrature formulae of Chebyshev type

The computation of sextic fields with a cubic subfield and no quadratic subfield . . . . . . . . . . . . . 419

See: Gatermann, Karin, Hoffmann, Christoph \& OpFer,

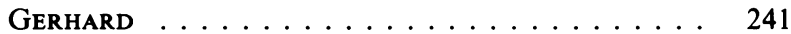

See: Hong, Y. P. \& PaN, C.-T. . . . . . . . . . . . . . 213

See: Bramble, James H. \& Pasciak, Joseph E. . . . . . 467

See: Di Natale, M., Gotusso, L., Pavani, R. \& Roux, D. 737.

See: Costabel, M., Penzel, F. \& Schneider, R. . . . . 575

On the distribution of quadratic residues and nonresidues modulo a prime number . . . . . . . . . . . 433

Efficient algorithms for periodic Hermite spline interpolation . . . . . . . . . . . . . . . 693

The discontinuous Galerkin method with diffusion . . . . 631

See: de Boor, CARL \& Ron, AMos . . . . . . . . . . . 705

See: Di Natale, M., Gotusso, L., Pavani, R. \& Roux, D. 737

Reconstruction techniques for classical inverse SturmLiouville problems . . . . . . . . . . . . . 161

See: Crisci, M. R., Russo, E. \& Vecchio, A. . . . . . . . 119

See: Rundell, William \& Sacks, Paul E. . . . . . . . 161

Krylov approximations for matrix square roots in stiff boundary value problems . . . . . . . . . . . 191

See: Costabel, M., Penzel, F. \& Schneider, R. . . . . 575

See: van der Geer, Gerard, Schoof, René \& VAN Der

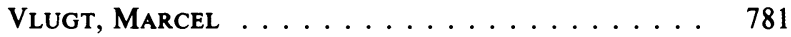

Higher-dimensional nonnested multigrid methods . . . . 457

Searching for primitive roots in finite fields . . . . . . . 369

See: Kechkar, Nasserdine \& Silvester, David . . . . 1

Computations of Siegel modular forms of genus two . . . 381

See: van Der Houwen, P. J., Sommeijer, B. P. \& Couzy,

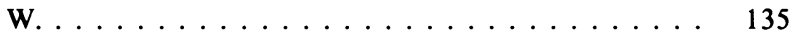

See: Chui, C. K., Stöckler, J. \& WARD, J. D. . . . . . . 255 
Author

Strain, JohN

TAsche, $M$.

TEMME, N. M.

Thomé, $\mathrm{V}$.

Traub, J. F. \&

WoźNIAKOWSKI, H.

VASSILEVSKI, P. S.

Vecchio, A.

VAN DER Vlugt, Marcel

WAHLBIN, L. B.

WALKINGTON, NoEl J.

WARD, J. D.

WASILKOWSKI, G. W. \&

GAO, F.

WoźNIAKowsKI, H.

Yeung, Man-Chung

ZHANG, SHANGYOU
Title Page

A fast Laplace transform based on Laguerre functions . . . 275 See: Plonka, G. \& Tasche, M. . . . . . . . . . . . 693

Asymptotic inversion of incomplete gamma functions . . 755

See: Chen, C., Thomee, V. \& Wahlbin, L. B. . . . . . . . 587

The Monte Carlo algorithm with a pseudorandom generator 323

Hybrid $V$-cycle algebraic multilevel preconditioners . . . 489

See: Crisci, M. R., Russo, E. \& Vecchio, A. . . . . . . . 119

See: van Der GeER, Gerard, Schoof, Rene \& VAN DER Vlugt, Marcel ................ 781

See: Chen, C., Thomee, V. \& Wahlbin, L. B. . . . . . 587

Convergence of nonconforming finite element approximations to first-order linear hyperbolic equations . . . . 671

See: Chui, C. K., Stöckler, J. \& WARd, J. D. . . . . . . 255

On the power of adaptive information for functions with

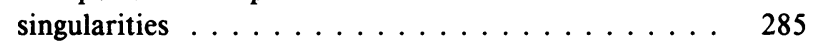

See: Traub, J. F. \& Woźniakowski, H. . . . . . . . . . 323

See: Chan, Raymond H. \& Yeung, Man-Chung . . . . . 233

See: Scott, L. Ridgway \& Zhang, Shangyou . . . . . 457 